\title{
Apego al método de Ponseti por parte de los familiares como determinante en el éxito del tratamiento del pie equinovaro
}

\author{
Adherence to the Ponseti method by family members as a \\ determinant in the success of club foot treatment \\ Fortis-Olmedo IO,* Ortiz-De Montellano-Gallaga MJ, ${ }^{\ddagger}$ \\ Altamirano-Duarte E, ${ }^{\ddagger}$ Martínez-Enríquez MJ, ${ }^{\ddagger}$ Ardón-Dubón JJ ${ }^{\ddagger}$ \\ Hospital Shriners para Niños, Ciudad de México.
}

RESUMEN. El pie equinovaro aducto congénito (PEVAC) es uno de los padecimientos más comunes en Ortopedia Pediátrica, el cual se compone del equino del retropié, el varo subastragalino, el aducto del antepié y el cavo del mediopié. El método de Ponseti es el tratamiento universalmente aceptado para el PEVAC; éste consta de tres fases: manipulación y enyesado, tenotomía del tendón de Aquiles y una fase de mantenimiento. El mayor porcentaje de las recaídas se presenta en la fase de mantenimiento; en la mayoría de los casos, está asociado el apego familiar deficiente. Presentamos el caso clínico de un paciente con PEVAC típico, tratado en cuatro ocasiones con el método de Ponseti y que no requirió tratamiento quirúrgico.

Palabras clave: Pie equinovaro, Ponseti, recidivante, tratamiento.

\section{Introducción}

El pie equinovaro aducto congénito (PEVAC), también conocido como "pie zambo», es uno de los padecimientos que más comúnmente son atendidos por el Servicio Ortopedia Pediátrica. Este padecimiento se define como la mala alineación congénita y compleja de los huesos del pie y del tobillo, junto con la displasia de todos los tejidos musculoesqueléticos distales a la rodilla. ${ }^{1}$

El PEVAC se caracteriza por presentar las siguientes cuatro deformidades principales: el equino del retropié, el varo subastragalino, el aducto del antepié y el cavo del mediopié (ocasionado por la flexión plantar del mediopié). ${ }^{1}$ Estas defor-
ABSTRACT. The clubfoot is one of the most common conditions in Pediatric Orthopedics, may affect each part of the foot and ankle, equinus, varus, and internal rotation of the calcaneum, and true equinus of the ankle are common. The Ponseti method is an universally accepted treatment, consisting of three phases: manipulation and plaster, Achilles tendon tenotomy and maintenance phase. The highest percentage of relapses occurs in the maintenance phase and the deficient family member is associated in most cases. We present a clinical case of a patient with typical clubfoot treated with the Ponseti method on four occasions without the need of surgical treatment.

Keywords: Clubfoot, Ponseti, recurrent, treatment.

midades son ocasionadas por alteraciones en los componentes óseos y los componentes blandos. Dentro de los componentes óseos, se encuentran la subluxación astrágalo-escafoidea, el sustentaculum tali subdesarrollado y la deformidad de la articulación calcáneo-cuboidea (desplazada medialmente). ${ }^{2} \mathrm{La}$ hipertrofia de ligamento deltoideo, el astrágalo-escafoideo y el tibial posterior más las alteraciones en la longitud de los músculos peroneos son las alteraciones de tejidos blandos más frecuentemente observadas en el PEVAC.,2

En el mundo, el PEVAC afecta aproximadamente entre 150,000 a 200,000 recién nacidos vivos. ${ }^{1}$ Su prevalencia mundial es de 1.2 casos por cada 1,000 recién nacidos vivos; en México, esta prevalencia es de 2.3 por cada 1,000

* Cirujano Ortopedista. Hospital Shriners para Niños, Ciudad de México.

${ }^{\ddagger}$ Cirujano Ortopedista. Nuevo Sanatorio Durango, Ciudad de México.
Dirección para correspondencia: Dr. Ignacio Osvaldo Fortis Olmedo E-mail: ignaciofortis@gmail.com 
recién nacidos vivos. ${ }^{3}$ El género masculino es el más afectado a nivel mundial (de dos a seis hombres y una mujer). ${ }^{3,4}$ Hasta en 50\% de los casos, el PEVAC puede presentarse de forma bilateral, sin embargo, cuando es unilateral, el lado derecho es el pie que resulta mayormente afectado. ${ }^{3}$ Existe una probabilidad de $4 \%$ de presentar PEVAC si uno de los padres tiene el antecedente de PEVAC y, si ambos padres padecieron esta patología, este porcentaje aumenta hasta $30 \%{ }^{4}$

Como tendencia actual, el tratamiento del PEVAC típico es conservador y el método de Ponseti es el más aceptado universalmente, lo que deja que el manejo quirúrgico se emplee para casos seleccionados de PEVAC atípico, en los cuales los pies son más complejos y más difíciles de manipular, o bien, presentan altas tasas de recidivas. ${ }^{5} \mathrm{Al}$ respecto, este método consta de las siguientes tres fases: la primera de ellas es la fase de manipulación y enyesado, que requiere en promedio el uso de cinco yesos colocados de forma semanal. La segunda fase consiste en la tenotomía del tendón de Aquiles y, finalmente, la tercera fase es la de mantenimiento con el uso de la barra de Dennis Brown. ${ }^{1}$

Bien aplicado, el método de Ponseti es efectivo y alcanza un éxito mayor a $90 \%$ de los casos. ${ }^{1}$ El apego estricto a este método, sobre todo en la fase final del tratamiento, resulta primordial, ya que es la base del resultado final de la patología. Así, ciertos elementos como el bajo estatus socioeconómico pueden constituir factores de riesgo para que el método obtenga malos resultados (a diferencia de la hiperlaxitud ligamentaria, que actúa como un factor protector). ${ }^{6}$

Hay que dedicarle una atención especial al manejo después de la corrección inicial en la etapa de mantenimiento, debido a que en ella se encuentra el mayor índice de recaída de esta patología. Posteriormente, con el manejo a través del método de Ponseti, puede haber deformidades residuales, entre ellas el equino (el más común), que se observa de 20 a $30 \%$ de los casos. ${ }^{7,8}$

Hasta el momento, han sido descritos cinco patrones de recaída, a saber: la disminución de la dorsiflexión (menor a
15 grados), supinación del antepié, el equino rígido, la abducción rígida del antepié y del mediopié y la combinación de éstos. ${ }^{8}$

\section{Caso clínico}

Presentamos el caso clínico de un varón de cinco años, con diagnóstico de pie equinovaro aducto congénito bilateral «típico», quien inició su tratamiento con método de Ponseti a la edad de cinco meses en nuestro hospital. A su llegada, presentaba un puntaje de 6 en la escala de Pirani y un Dimeglio de IV bilateral (Figura 1). Se colocaron ocho yesos de Ponseti de forma semanal con corrección de todas las deformidades y, a la edad de siete meses, se realizó una tenotomía bilateral del tendón de Aquiles, con posterior uso de yesos de Ponseti durante tres semanas más.

Después del retiro de los yesos, inició la fase de mantenimiento con el uso de la barra de Dennis Brown «23 horas por día». A la edad de 11 meses, presentó recidiva de todas las deformidades, con un puntaje de 4 en la escala de Pirani y Dimeglio III bilateral. Por lo anterior, se reinició un segundo ciclo de yesos de Ponseti con tres manipulaciones y enyesados de forma semanal; al año de edad, al paciente se le realizó la primera revisión de la tenotomía bilateral del tendón de Aquiles. Al término de las tres semanas de uso de yesos de Ponseti, se reinició el uso de la barra de Dennis Brown «23 horas por día»; sin embargo, en las consultas subsecuentes ya no se tuvo la certeza del adecuado uso de la barra por parte de los familiares.

$\mathrm{Al}$ año y tres meses de edad, presentó una segunda recidiva de las deformidades de ambos pies, cuyo puntaje de Pirani fue de 4 y Dimeglio III bilateral. Seguido de lo anterior, se optó por reiniciar el tercer ciclo de manipulación y enyesado; sin embargo, al ser un niño de mayor edad y al ofrecer resistencia para su manipulación, se decidió llevar a cabo el tratamiento con sedación, con una periodicidad de dos semanas entre yeso y yeso. Posterior a los cuatro yesos de Ponseti cuando el paciente tenía una edad de un año y cinco meses, se le realizó una segunda revisión de la
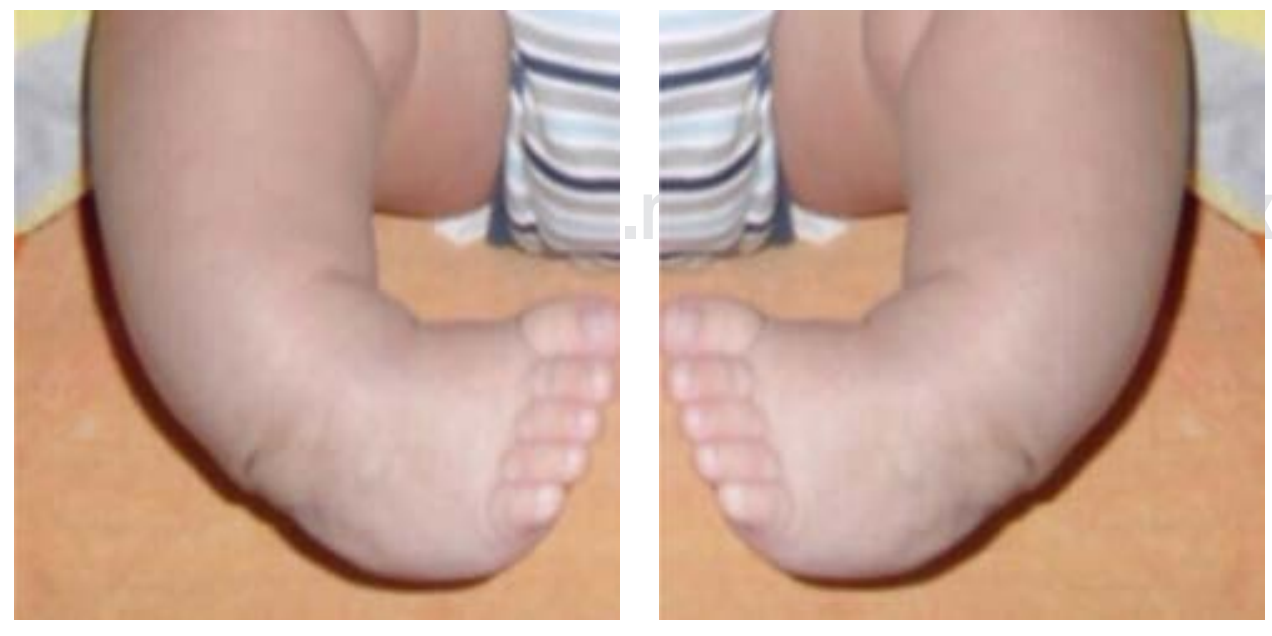

Figura 1:

Deformidad inicial a los cinco meses de edad. 

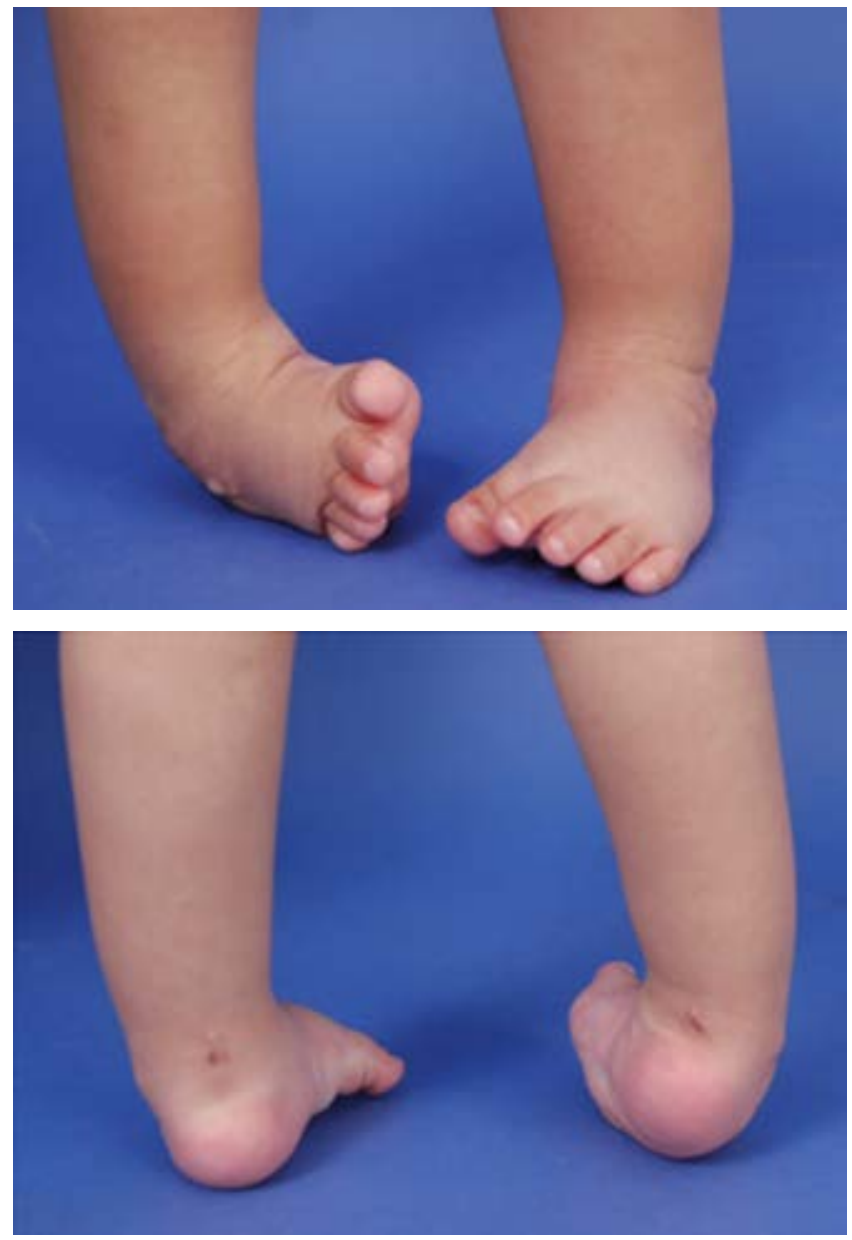

Figura 2: Deformidad residual al año, 10 meses de edad.

tenotomía bilateral del tendón de Aquiles con posterior reinicio de la barra abductora «23 horas por día». No obstante, de nuevo no contamos con la certeza absoluta del apego adecuado del paciente y los familiares al tratamiento con la barra abductora.

A la edad de un año y 10 meses de edad, el paciente, otra vez, presentó una nueva recidiva de todas las deformidades, con un puntaje de 3 en la escala de Pirani para el pie derecho y 2.5 para el pie izquierdo y un Dimeglio de III de forma bilateral (Figura 2). Al enfrentarnos a un PEVAC «típico» con corrección de todas las deformidades durante la fase de manipulación-enyesado y ante unos familiares que aseguraron un "correcto» uso de la barra abductora, solicitamos estudios de rayos $\mathrm{X}$ y resonancia magnética de columna (Figura 3) y descartamos la posibilidad de un PEVAC neurológico (diastematomielia, síndrome de cordón anclado, diplomelia, etcétera). Finalmente, se optó por reiniciar por cuarta ocasión el método de Ponseti; requerimos cinco sesiones de manipulación-enyesado quincenales bajo sedación, con corrección de todas las deformidades. Al término, se le realizó una tercera revisión de la tenotomía bilateral del tendón de Aquiles, y se hizo hincapié a los familiares sobre la importancia de su papel en el tra- tamiento para lograr un resultado exitoso. Posteriormente, se reinició de nuevo el uso de la barra abductora 23 horas al día hasta la edad de dos años y cinco meses. Después pasó a «16 horas por día» hasta la edad de tres años y seis meses, para concluir con «12 horas por día» hasta la edad de cinco años.

Actualmente, el paciente se encuentra en vigilancia estrecha cada tres meses sin presentar recidivas de las deformidades (Figura 4).

\section{Discusión}

Se define como «recurrencia» a la reaparición de cualquiera de los componentes de la deformidad del PEVAC después del tratamiento inicial y que requiera tratamiento adicional y/o intervención quirúrgica para resolverla. ${ }^{9}$ De acuerdo con la literatura, la recurrencia de las deformidades asociadas con el pie equinovaro aducto congénito idiopático se presenta entre 20 y $30 \%$ de los pacientes sometidos al tratamiento; en este punto, el seguimiento continuo de los niños en tratamiento es esencial para identificar y tratar las recurrencias de manera oportuna. ${ }^{10}$

El caso clínico presentado en este trabajo mostró una recurrencia de la mayoría de las deformidades del PEVAC en tres ocasiones; ello ocurrió después de un tratamiento adecuado, lo que requirió el reinicio de la fase de enyesado y tenotomía de revisión en tres ocasiones más. Sin embargo, al presentar reaparición de las deformidades, se supuso que el incumplimiento en la fase de mantenimiento con el uso de la barra de Denis Brown era el principal determinante en el resultado exitoso del tratamiento. Luego de descartar un PEVAC neurológico y de orientar y sensibilizar a la familia
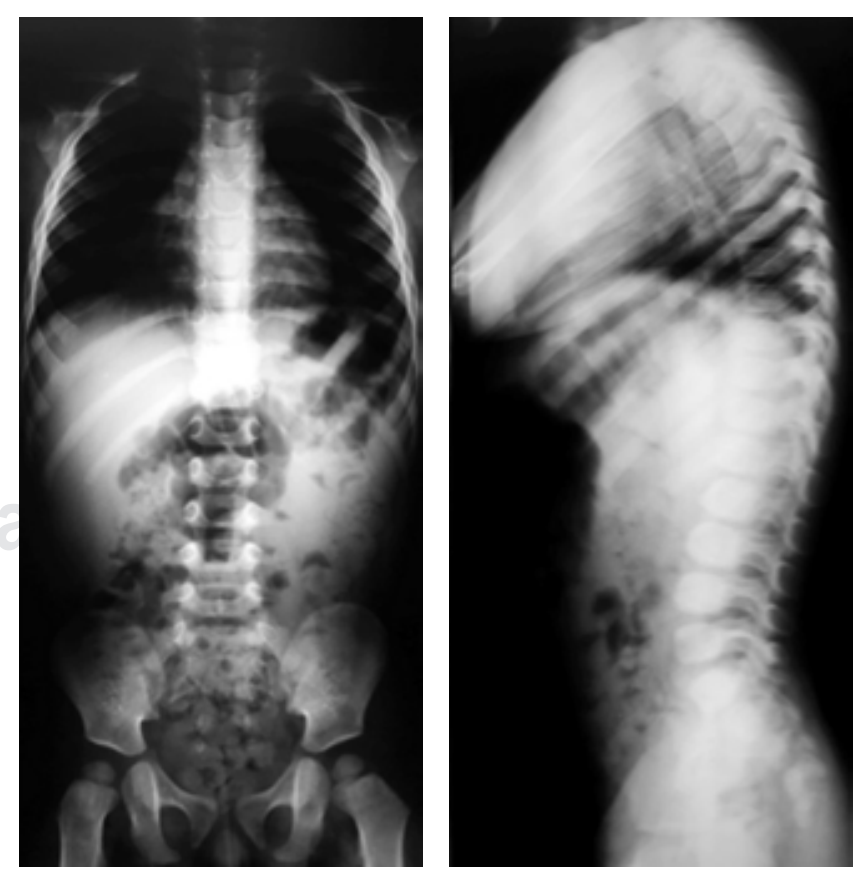

Figura 3: Radiografías de columna en proyección anteroposterior y lateral. 

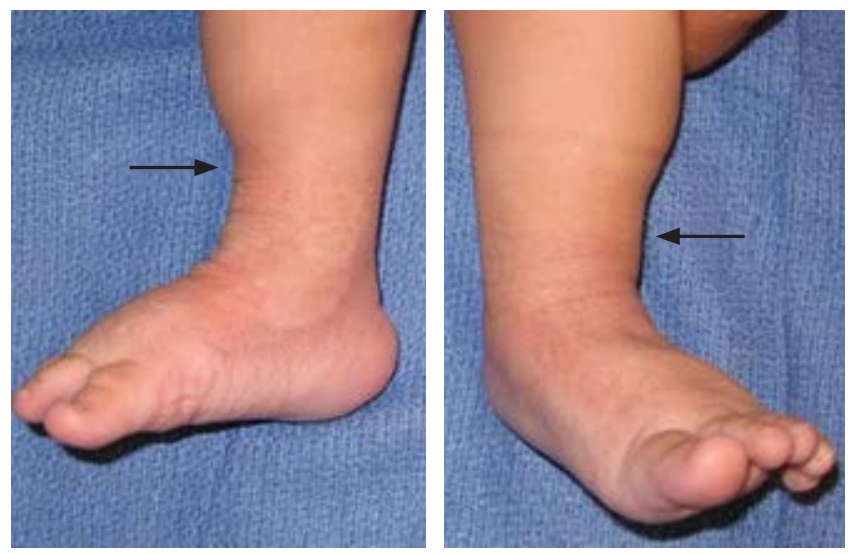

Figura 4: Se observa signo de la familia obediente (flechas).

del paciente sobre la importancia de su papel en el tratamiento de la patología de su hijo, se observó una evolución favorable y ausencia de deformidades residuales en las consultas de seguimiento.

Existen tres causas principales de recidivas en el PEVAC típico: tratamiento inadecuado, pie neurológico y mal apego al tratamiento de mantenimiento; ${ }^{9}$ este último es el que ocupa la mayor parte del porcentaje. Estudios realizados por Morcuende han demostrado que las recidivas ocurren en $6 \%$ de familias que cumplen el tratamiento correctamente, en comparación con $80 \%$ de familias que no los cumplen. Contrastado con la literatura internacional, el fracaso en el tratamiento de nuestro paciente fue atribuido al mal apego familiar en la fase de mantenimiento.

Por ello, se concluye que el apego y compromiso de la familia de un paciente con PEVAC que se encuentra en tratamiento determinará en la mayoría de las ocasiones su éxito. El seguimiento continuo de los niños con PEVAC es esencial para identificar y tratar las recurrencias de manera oportuna.

Bibliografía

1. Staheli L. Pie Zambo: El método de Ponseti. 3a. ed. Global HELP Organization; 2009.

2. Foster A, Davis N. Congenital tlipes equinovarus. Surgery. 2007; 25: 171-5.

3. Torres-Gómez A, Pérez-Salazar-Marina D, Cassis N. Pie equino varo aducto congénito, prevalencia en una población mexicana. Rev Mex Ortop Pediatr. 2010; 12(1): 15-8.

4. Roye D, Roye B. Idiopathic congenital talipes equinovarus. JAAOS. 2002; 10(4): 239-48.

5. Balasankar G, Luximon A, Al-Jumaily A. Current conservative management and classification of club foot: a review. J Pediatr Rehabil Med. 2016; 9(4): 257-64.

6. Cosma DI, Corbu A, Viorel ND, Todor A, Valeanu M, Morcuende J, Man S. Joint hyperlaxity prevents relapses in clubfeet treated by Ponseti method-preliminary results. Int Orthop. 2018; 42(10): 243742. doi: 10.1007/s00264-018-3934-7.

7. Ponseti International Association [portal web]. What is clubfoot? Disponible en: http://www.ponseti.info/that-is-clubfoot.html.

8. Mitchell J, Bishop A, Feng Y, Farley D, Hetzel S, Ploeg HL, et al. Residual equinus after the Ponseti method: an MRI-based 3-dimensional analysis. J Pediatr Orthop. 2018; 38(5): e271-7. doi: 10.1097/BPO.0000000000001147.

9. Uglow M, Kurup H. Residual clubfoot in children. Foot Ankle Clin Am. 2010; 15: 245-264.

10. Docquier PL, Leemrijse T, Rombouts JJ. Clinical and radiographic features of operatively treated stiff clubfeet after skeletal maturity: etiology of the deformities and how to prevent them. Foot Ankle Int. 2006; 27(1): 29-37. 